\title{
The Inquiring System Based on Wechat for the Instruction Information of Class
}

\author{
Chunfen $\mathrm{Bu}^{1, \mathrm{a}^{*}}$ \\ ${ }^{1}$ Department of physical science and technology, Kunming University \\ a18459423@qq.com \\ *The Corresponding Author
}

Keywords: Instruction technology; WeChat; Self-service inquiry system

\begin{abstract}
Now the Tencent Inc launched WeChat application has become the majority of mobile phone users to install higher use rate of high school students, especially the very widespread application. WeChat public platform is the foundation of the Tencent Inc in WeChat add function module of an extended, through this platform to develop a class of self-service information query system APP, you only need to install WeChat in mobile phone, pay attention to a particular WeChat public account, do not have to consider mobile phone system version the problem, fast and convenient access to all kinds of class information, can also carry out independent query.
\end{abstract}

\section{Introduction}

In today's college life, students communicate with the teacher in charge of the rare, the student to the class information sources more and more along with the development of the Internet age, the student to the use of the Internet is becoming more and more demand for at any time and anywhere more and more strong. With the development and progress of network technology and modernization speed up the pace of information construction, the domestic many universities most established a relatively perfect information system. Through the system between teachers and students can get all kinds of information more convenient. In today's college life, students communicate with the teacher in charge of the rare, the student to the class information sources more and more along with the development of the Internet age, the student to the use of the Internet is becoming more and more demand for at any time and anywhere more and more strong. With the development and progress of network technology and modernization speed up the pace of information construction, the domestic many universities most established a relatively perfect information system. Through the system between teachers and students can get all kinds of information more convenient.

WeChat is tencent launched a mobile communications software, support sent voice messages through mobile phone network, video, images and text, can be single and group chat, also can be found according to their location near the people, bring brand-new communication to the user experience.

In this paper, WeChat public platform for the students of class information self-service inquiry system, can more easily query the student personal information and schedule information, the test time and performance information, input relevant keyword query and information to students with text, images, video, voice categories. The teacher in charge, that is, the system administrator from the background operation in a timely manner to add the student's schedule information, the test information and performance information, and modify as needed. So that students can query need to record information anytime and anywhere, schedule information, the test time, etc. Changed a single model of information query. 


\section{Method}

Account registration:

Log on to https:// mp.weixin.qq.com/ or in each big search engine website login "WeChat public platform", click "register now" to complete the basic information registration according to clew.

Activation: basic information to fill in, after the completion of steps in the basic information to fill in the email will receive an activation email, click on the link within the email address can be activated, and then login WeChat public platform for other information registration can be completed WeChat registration of public platform.

Audits: information registration is completed, the general need to 7 working days of waiting for audit, the audit before success, the public can only realize the basic function, cannot achieve mass, some functions such as secondary development.

Account: WeChat public platform account not to add buddy users, can only be added as a friend, you can through the qr code scans or search WeChat ID way, students can add number the headteacher WeChat public attention to implement the message interaction. Teacher can put the WeChat public or qr code information to other ways such as QQ, weibo told the students, in order to realize the platform promotion.

\section{Design}

The structure of our system is given as follows:

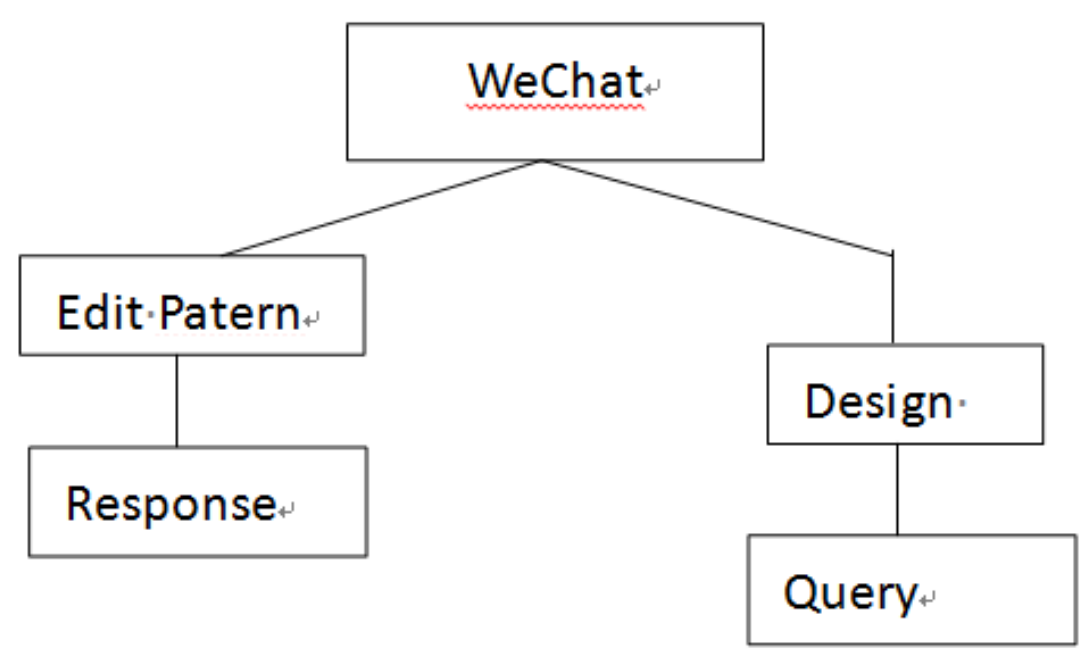

Figure 1. Structure of design

Based on PHP development as an example, this paper shows the interface development process. The student self-service query WeChat public interface development platform, mainly including authentication interface and query interface.

Authentication interface is mainly in order to enable interface, realize WeChat docking platform authentication function, when validation WeChat public platform will send a get request to the interface address, verify the interface by requesting access to the three parameters, and then validated algorithms in accordance with the contract, through feedback after token value of the agreement, to ensure that a valid url.

\section{The System Showing}

\section{Apply for Message Interface}

To access the website platform developers WeChat public access in the guide to download the official sample code, get wx_sample. PHP interface program, it is changed to wx, PHP, and modify 
the source code, to define (" TOKEN ", "weixin") is modified to define (" TOKEN ", "FTCX"), and FTCX here is TOKEN, downloaded to the website of http://wx.ft.cn/, a root directory (see the appendix) PHP source code.

After entering WeChat public platform, and then click the "advanced features - > development model $->$ become a developer", then fill in the order processing interface application URL (requirement must begin with http://, and supports only port 80, for example, http://wx.ft.cn/wx.php) and Token (must be Numbers or English, such as FTCX), then click submit, if prompt "has become a developer, that successful application interface.

\section{Codes}

The part of our system is listed as follows:

$\langle x m l>$

$<$ ToUserName $><![C D A T A[\$$ fromUsername]] $></$ TouserName $\rangle$

$\langle$ FromeUserName $\rangle\langle$ ![CDATA[\$toUsername]] $>\langle/$ FromeUserName $\rangle$

$<$ CreateTime $>\$$ CreateTime $</$ CreateTime $>$

$<$ MsgType $\rangle\langle$ ! [CDATA[text] $\rangle\langle/$ Msgtype $\rangle$

$<$ Content $><![C D A T A[\$$ Content]] $></$ content $>$

$<M s g I d>1234567890123456</ M s g I d>$

$</ x m l>$

\section{Functions}

WeChat platform are used to achieve the following basic functions:

Management:

Real-time news WeChat public platform itself provides text, images, voice, video, graphic information such as real-time interactive features, complete with a specified message interaction of students.

The group-sent message: the specified students send text, images, voice, video, graphic information, such as information push in batches. Such as the test time arrangement, class time adjusting batch delivery information such as news.

User management: the students to focus on news management, including manual authentication, grouping, modification note, etc.

Results

The system when it is executed is given as follows:

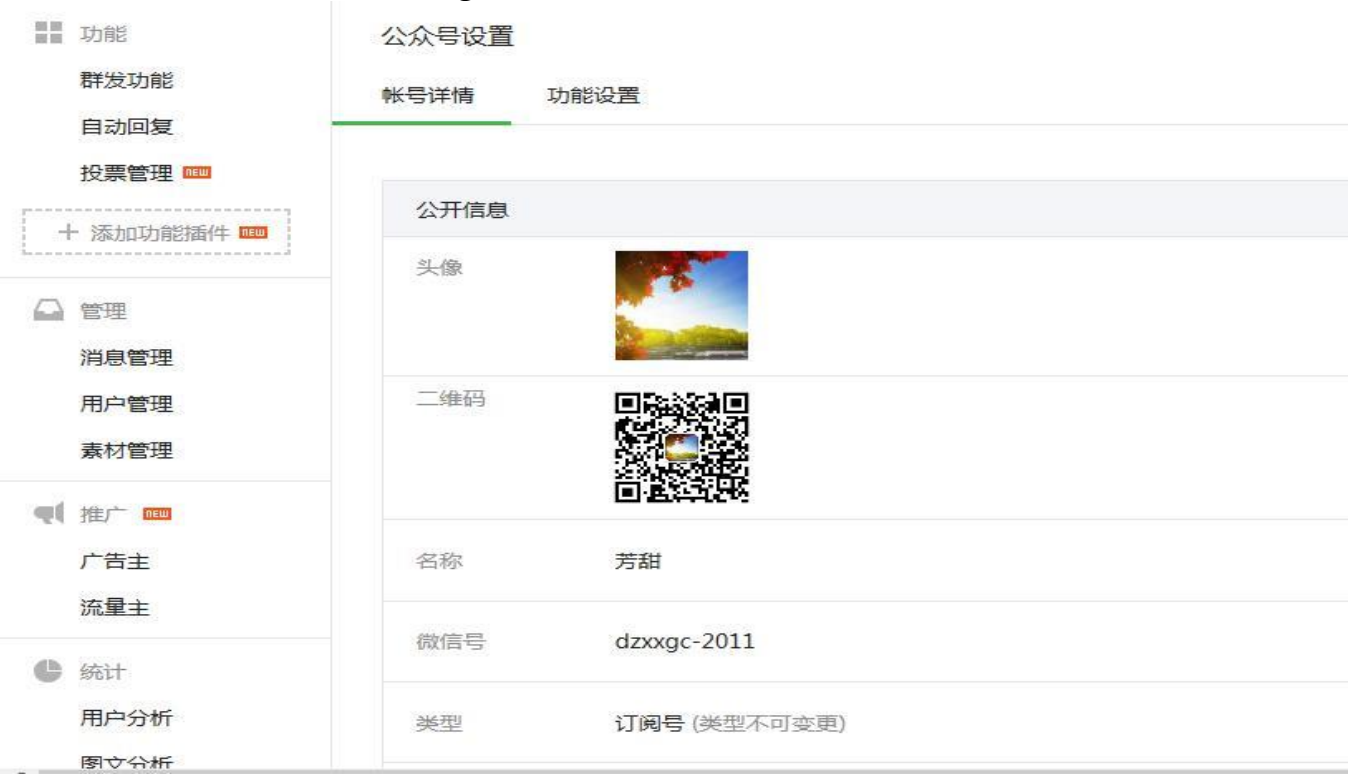

Figure 2. The results of our system 


\section{Conclusion}

This paper implemented a WeChat based public account inquiry information to the class and grade self-service system, using a mobile phone WeChat client queries you need the test information, results, schedule information, the test time, etc. In this article, through secondary development WeChat public platform, which can realize the class and students' management, to form the corresponding user groups, two-way form of multimedia information exchange and interaction can be realized. More important is the use of secondary development platform, implementing the self-service query of all kinds of class information.

\section{Reference}

[1] J. Rissanen, A universal data compression system, IEEE Trans. Inform. Theory, vol. 29, pp. 656-664, Sept. 1983.

[2] J. Rissanen, Strong optimality of the normalized ML models as universal codes and information in data, IEEE Trans. on Information Theory, vol.IT-47, No. 5, pp.1712-1717, 2001.

[3] S.Forchhammer, X.Wu, J.D.Andersen, Optimal context quantization in lossless compression of image data sequences, IEEE Transactions on Image Processing 13(4), pp.509-517, Apr. 2004.

[4] S.Forchhammer, X.Wu, Context quantization by minimum adaptive code length, in: Proc. of IEEE Inter. Symposium on Information Theory, Nice, France, pp.246-250, June 2007.

[5] Min Chen, Jianhua Chen, Affinity propagation for the Context quantization, Advanced Materials Research, Vols. 791, pp.1533-1536, 2013.

[6] Enhancing Teaching and Learning through Education Data Mining and Learning Analytics[J], Education Department of America, pp.336-339, 2012.

[7] Bapler.P\&Murdoch, Academic Analytics on Data Mining in Higher Education. Intemational Jounlal for the Scholarship of Teaching and Leanling, Vol.4(2), pp.1926-1933.2013. 